\title{
Awake ECMO without anticoagulation for nearly completely obstructive squamous cell carcinoma of the trachea
}

\author{
Theodore D. Klug $^{1}$ (D) | John Craig ${ }^{2}$ | Courtney B. Shires ${ }^{1}$
}

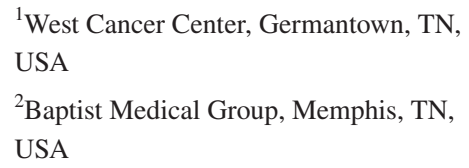

${ }^{1}$ West Cancer Center, Germantown, TN, USA

${ }^{2}$ Baptist Medical Group, Memphis, TN, USA

\section{Correspondence}

Theodore D. Klug, West Cancer Center, 7945 Wolf River Blvd, Germantown, 38104 TN, USA.

Email: theodklu@gmail.com

\begin{abstract}
Awake veno-venous ECMO without anticoagulation is a safe and successful way to remove a significantly obstructive malignancy in the trachea.
\end{abstract}

\section{K E Y W O R D S}

anticoagulation, complications, ECMO, trachea

\section{INTRODUCTION}

The objective of this case report was to describe the operation and use of awake ECMO without anticoagulation in a patient with a nearly completely obstructive squamous cell carcinoma of the trachea. A retrospective chart and literature review were performed in a private hospital in a large municipal area.

Veno-venous extracorporeal membrane oxygenation (ECMO) is now being applied to spontaneously breathing, awake patients when traditional, mechanical ventilation is not possible. ${ }^{1}$ Current ECMO therapies provide a variety of options for the multidisciplinary teams involved in management. ${ }^{2}$ Veno-venous ECMO can provide complete respiratory support, however, with substantial risks, including bleeding, thromboembolic events, and infection. ${ }^{2}$ Still, the advantage of veno-venous ECMO on awake patients minimizes the adverse effects of mechanical ventilation, including barotrauma, hypotension, and arrhythmias. ${ }^{3,4}$ It also minimizes the duration of neuromuscular blockade use that is usually used to improve patient ventilator dyssynchrony. ${ }^{3,5}$ This method decreases the risk of critical care associated polyneuromyopathy and delirium as well. ${ }^{3}$

The decision was made to not use anticoagulation during the procedure. With thromboembolic complications remaining the major causes of death in patients undergoing ECMO treatments, this decision was unique and a crucial component of our particular procedure. ${ }^{6}$ With complications commencing upon blood contact with artificial surfaces of the circuit, blood pump, and oxygenator system, ECMO use naturally increases the risk of hemorrhagic and thromboembolic events. ${ }^{6}$ Therefore, anticoagulation therapy, predominantly with unfractionated heparin, is almost always required in cases to prevent these problems. ${ }^{6}$ Still, with a lack of high-quality data to guide anticoagulation management in ECMO patients, results in marked practice vary considerably among centers. ${ }^{7}$ In our particular case, increased risk of bleeding and small area of operating site were taken into consideration, therefore leading to the decision to not use anticoagulation. Although highly effective at the prevention and treatment of thromboembolism, anticoagulants are by their very nature associated with significant bleeding risks. ${ }^{8}$ Numerous individual clinical factors have been linked to an increased risk of hemorrhage, with our particular patient being susceptible, given her older age and history of renal disease. ${ }^{8}$ More specific details of the patient and Otolaryngology procedure will follow.

\section{$2 \mid$ METHODS}

This retrospective chart review included one patient that underwent awake veno-venous ECMO without anticoagulation 
for completely obstructive SCC by a single Cardiothoracic Surgeon and Otolaryngologist at a large private hospital in January of 2020. Demographics and preoperative risks were collected. Data were gathered regarding the type of procedure performed. Postoperative variables and imaging findings were recorded. A literature review was then performed to discuss the use of ECMO in this particular procedure, along with the decision to not anticoagulate.

\section{3 | RESULTS}

A 71-year-old woman initially presented in January of 2017 to an outpatient clinic in Memphis, TN, with a base of tongue mass. Her past medical history included hypertension (HTN), transient ischemic attach (TIA), acute cerebrovascular accident (CVA), acute respiratory failure with hypoxia, obesity, history of breast cancer, mixed hyperlipidemia, shortness of breath (SOB), depression with anxiety, and a 50-year history of tobacco use. Family history included HTN in her deceased mother, a heart attack in her deceased father, and diabetes in her brother. There was no family history of cancer. She had no history of drug or alcohol use.

In March of 2017, she then presented to her PCP with upper respiratory infection (URI) symptoms, including cough, congestion, and sore throat. On 6 April 2017, she was admitted to Baptist East with stridor and shortness of breath (SOB). She had a CT performed, which showed a $2.4 \times 2.1 \times 2.2 \mathrm{~cm}$ enhancing polypoid mass at the base of tongue with some downward mass effect on the epiglottis. Just above the sternal notch and $5 \mathrm{~cm}$ inferior to the vocal cords, there was also a $10 \times 17 \times 14 \mathrm{~mm}$ polypoid lesion, causing moderate stenosis of the trachea (Figure 1). The patient then had a biopsy of the tracheal mass and received a tracheostomy. Pathology from the trachea showed squamous cell carcinoma. On April 7, she underwent a direct laryngoscopy and biopsy. The biopsy of the base of tongue was negative for malignancy.

The patient then had a PET scan on April 20, which showed increased FDG activity in the base of tongue $(\mathrm{L}>\mathrm{R})$ and around the tracheostomy site. She then underwent resection of her lingual tonsils on 8 May 2017. On August 1, she then underwent a tracheal resection procedure. Pathology of the trachea showed squamous cell carcinoma with negative margins.

She was lost to follow-up until 2020, when she presented to the same hospital with stridor. She no longer had the tracheotomy tube at that time and had been doing well by all accounts since 2017. According to the patient, she was eating an oral diet and had not coughed up any blood since 2017 . In January 2020, she was scheduled for laryngoscopy and bronchoscopy with possible dilation and biopsy. However, repeat imaging showed a $3 \times 2 \mathrm{~cm}$ tracheal mass with possible esophageal involvement. The tracheal lumen was also significantly narrowed. It was decided that intubation could

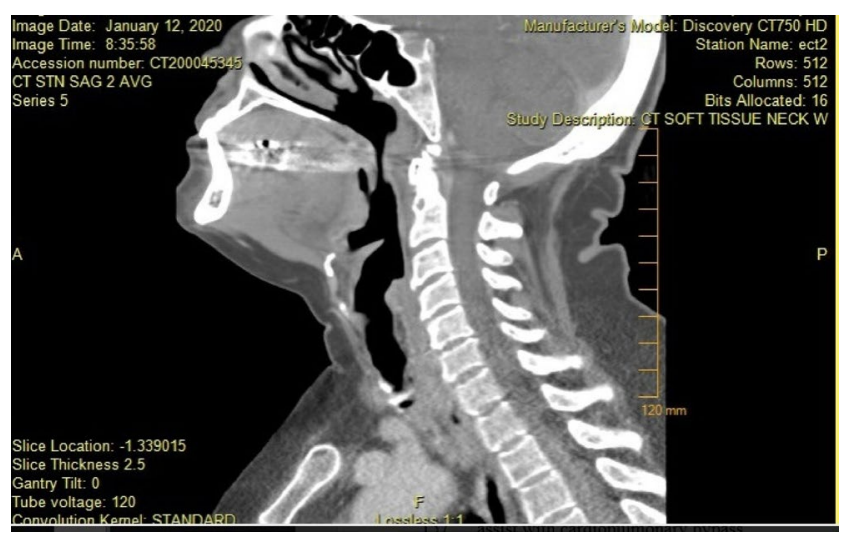

F I G URE 1 Sagittal view of the CT scan showing intraluminal tracheal mass

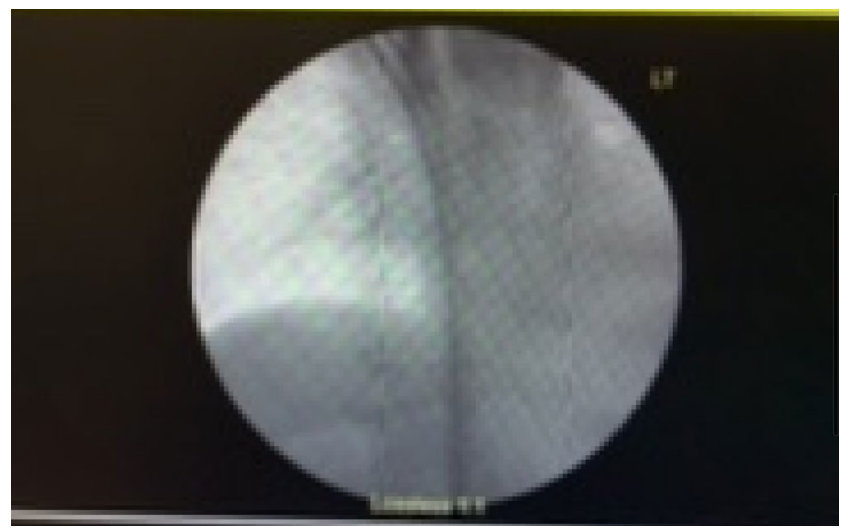

F I G URE 2 Radiograph of cannula in the right internal jugular vein that was advanced through the superior vena cava into the right atrium

not be performed due to the size of the mass. Therefore, cardiothoracic surgery was consulted to assist with cardiopulmonary bypass.

The patient was brought to the operating room on 15 January 2020 and placed supine on the operating table. Venovenous ECMO oxygenation was performed. In the classic configuration, veno-venous ECMO support is achieved with two cannulae, one usually inserted in the right femoral vein and advanced to the junction between the inferior vena cava (IVC) and the right atrium (RA) and the other inserted in the right internal jugular vein (IJV) and advanced through the superior vena cava (SVC) into the RA. ${ }^{2}$ This configuration was implemented in our patient procedure (Figure 2).

The patient was then prepped and draped for laryngoscopy and bronchoscopy. The laryngoscope was placed in the oral cavity, and a tooth block was used to protect dentition. The laryngoscope was suspended using a Lewy arm and advanced to view the larynx. A zero-degree telescope was then placed through the true vocal cords (Figure 3). The large tracheal mass was visualized, and several biopsies were taken with cup forceps (Figure 4). The mass seemed to originate from 
the left posterolateral wall of the trachea. The microdebrider was then placed in the trachea and used to suction the mass into the central lumen of the trachea, away from the lateral wall. The mass was debrided using the microdebrider. Ninety percent of the mass was removed, Afterward, Afrin-soaked pledges were used to provide hemostasis. Upon re-examination, the trachea was widely patent (Figure 5). The laryngoscope and telescope were then removed, and the patient was turned over to anesthesia. She was intubated orally and transferred to the ICU. On postoperative day 1 , she was extubated. She had no stridor and no respiratory complaints.

Final pathology showed high grade weakly keratinizing SCC with variable koilocytosis. Immunohistochemistry for p16 showed diffuse strong nuclear and cytoplasmic positive staining confirming HPV origin. The PD-L1 for Keytruda (Pembrolizumab) was positive with a combined positive score (CPS) of 15, representing the number of PD-L1staining cells divided by the total number of variable tumor cells and then multiplied by 100 . The PD-L1 for Opdivo was also positive.

Cisplatin chemotherapy and concomitant radiation were begun on February 5. CT performed on February 5 no longer showed a tracheal mass. PET showed multifocal increased FDG activity along the left posterior wall of the trachea just below the level of the thyroid gland and near the tracheal esophagus, concerning for neoplastic activity. There was also increased activity in the rectal cavity, concerning for neoplasia. Following completion of chemotherapy and radiation for her tracheal tumor, the patient then underwent a colonoscopy

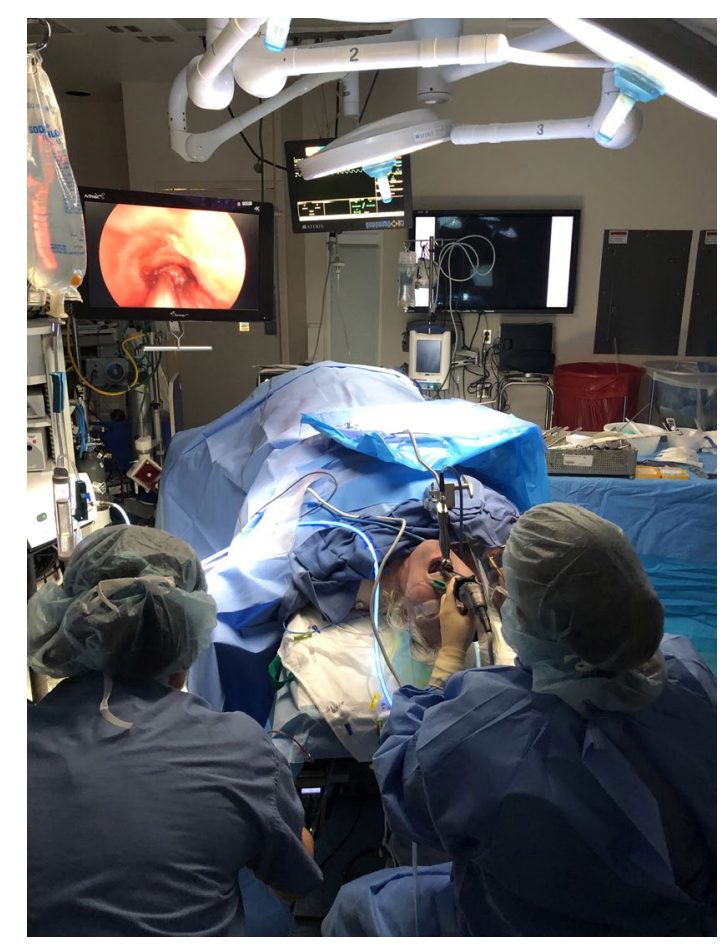

F I G URE 3 Operating room setup. The laryngoscope in suspension with a Lewy 6 arm. A zero-degree telescope placed through the laryngoscope and polypectomy. Final GI pathology from the nearby cecum showed colonic mucosa with benign, prominent submucosal lymphoid aggregate. There was no presence of adenoma or malignancy. Final pathology results from the rectum showed fragments of hyperplastic polyps with mucosal prolapse features, but no adenomas or malignancy.

Results of a post-treatment PET-CT were negative, except for some uptake in the rectal area, which proved more likely due to the previous polypectomy.

Provided good findings during her 3 June 2020, bronchoscopy, PEG tube removal can be considered.

\section{4 | DISCUSSION}

This patient underwent a resection of her trachea in 2017 for reportedly HPV-positive squamous cell carcinoma (SCC). She was then lost to follow-up and received no treatment

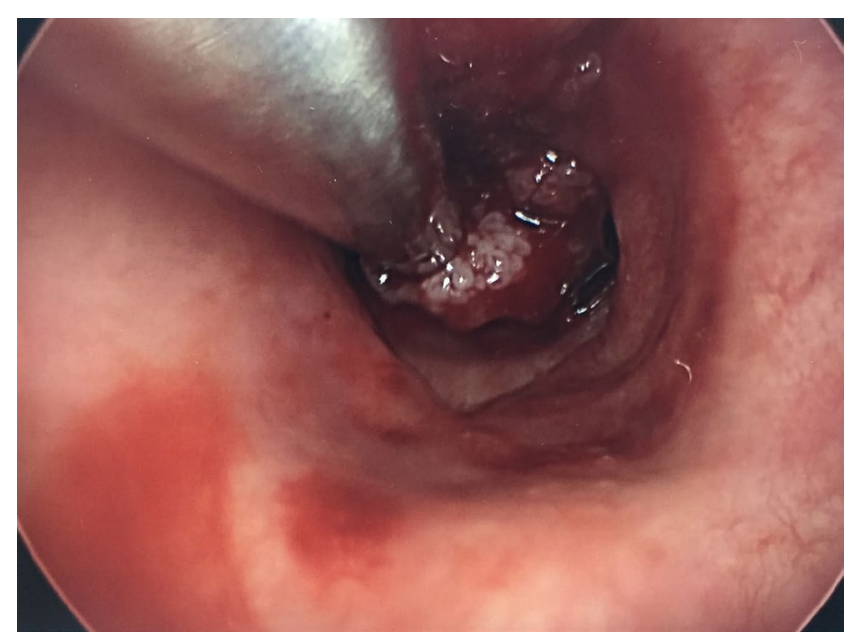

F I G URE 4 Endoscopic view of the large tracheal mass prior to biopsy or eight debridement

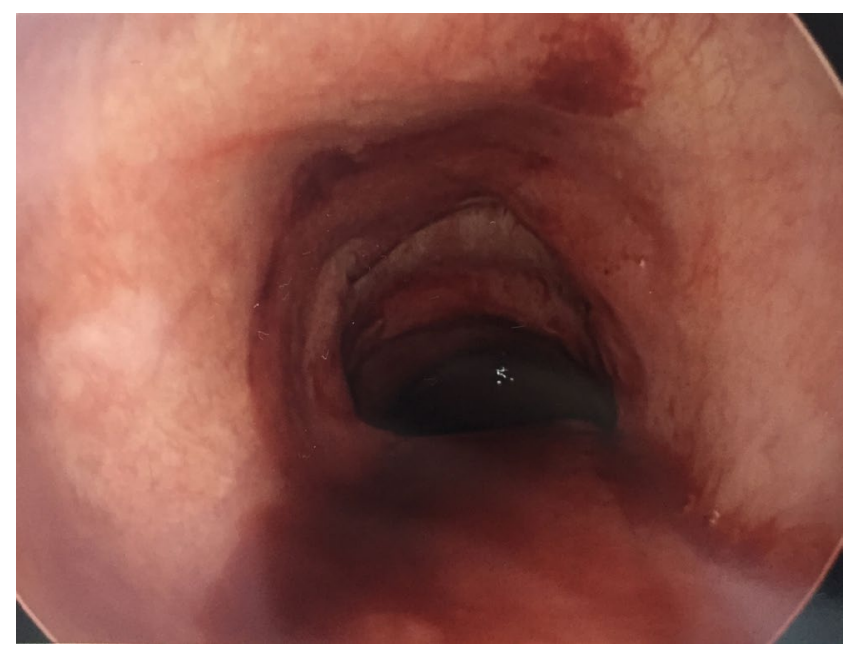

F I G U RE 5 Endoscopic view of the trachea after debridement of tracheal mass 
up until 2020, when she reappeared with stridor and a significantly obstructing tracheal mass just below the thoracic inlet, with apparent transmural invasion and involvement of at least the esophageal adventitia. In turn, mechanical ventilation could not be performed.

This SCC was a typical pattern HPV tumor with clear confirmation by diffuse nuclear cytoplasmic p16 immunohistochemical positivity. The PD-L1 for Keytruda (Pembrolizumab) was positive with a combined positive score (CPS) of 15. The PD-L1 for Opdivo was also positive. Pembrolizumab is a selective humanized IgG4 kappa monoclonal antibody that inhibits the programmed death-1 (PD-1) receptor, an integral component of immune checkpoint regulation in the tumor microenvironment. ${ }^{9}$ The PD-L1 and PD-L2 ligands on tumors can bind with PD-1 receptors on T cells to inactivate them. ${ }^{9}$ Pembrolizumab binds to the PD-1 receptor and blocks its interaction with PD-L1 and PD-L2, which helps to restore the immune response. ${ }^{9}$ The drug is currently approved by the Food and Drug Administration (FDA) for the treatment of metastatic or unresectable, recurrent head and neck SCC whose tumors express PD-L1 [CPS $>=1$ ], with disease progression on or after platinum-containing chemotherapy. It has also been approved for treatment of advanced melanoma and metastatic squamous and nonsquamous nonsmall cell lung cancer (NSCLC). ${ }^{9}$ Ultimately, according to a 2015 study by Bowman, the greatest value to be derived from tracking PD-L1 testing is the ability to link test results to treatment decisions. ${ }^{10}$

As of May 15, there has been no recurrence of the symptoms since surgery, and imaging shows a clear and patent trachea. The implications of PD-L1 staining in this patient and subsequent choice of chemotherapy will continue to be evaluated throughout the patient follow-up period.

Regarding the decision to not use any form of anticoagulation during the procedure, blood loss was minimal, less than $10 \mathrm{~mL}$. There also were no thrombotic or hemorrhagic complications during or after this surgery. Based on these positive results, we recommend that anticoagulation and its possible benefits and negative side effects be considered in full effect for any procedure dealing with a small opening in a head and neck procedure. For our group, the potentially catastrophic mid-procedure outcomes from use of anticoagulation caused us to decide against its use, especially given the very small opening through which the tracheal procedure occurred. In sum, the ultimate fear for our group was that intraoperative bleeding in the small operative space would lead to an inability to continue and successfully complete the operation. With limited visualization, the resection of 90 percent of the tracheal mass would not have been possible. As mentioned above for the PD-L1 staining, thrombotic and hemorrhagic events will continue to be at forefront of our minds during this postoperative and follow-up period. We will continue to monitor the patient and check for any changes or discrepancies in her $\mathrm{CBC}$, BMP, and clotting factors.

\section{CONCLUSION}

Awake veno-venous ECMO without anticoagulation is a safe and successful way to remove significantly obstructive malignancies in the trachea.

\section{ACKNOWLEDGMENTS}

None. Published with written consent of the patient.

\section{CONFLICTS OF INTEREST}

The authors report no relevant financial disclosures related to this current work.

\section{AUTHOR CONTRIBUTION}

CBS, MD, FACS, TK, MD, MPH, and JCraigMD, FACS: collected data, wrote, and edited article.

\section{ETHICAL APPROVAL}

All issues related to ethics were taken into consideration throughout the study design and proposal and implemented during the research study itself. Informed consent was obtained, beneficence was made a top priority, and respect for confidentiality and privacy were upheld during the study and its various analysis and information assertion components. The patient agreed to allow the authors to publish the case and included images.

\section{DATA AVAILABILITY STATEMENT}

Data are available upon request.

\section{ORCID}

Theodore D. Klug (D) https://orcid. org/0000-0002-2829-5846

\section{REFERENCES}

1. Crotti S, Bottino N, Spinelli E. Spontaneous breathing during veno-venous extracorporeal membrane oxygenation. J Thorac Dis. 2018;10(S5):S661-S669. https://doi.org/10.21037/jtd.2017.10.27.

2. Banfi C, Pozzi M, Siegenthaler N, et al. Veno-venous extracorporeal membrane oxygenation: cannulation techniques. J Thorac Dis. 2016;8(12):3762-3773. https://doi.org/10.21037/jtd.2016.12.88

3. Chacko CJ, Goyal S, Yusuff H. Awake extracorporeal membrane oxygenation patients expanding the horizons. J Thorac Dis. 2018;10(Suppl 18):S2215-S2216. https://doi.org/10.21037/ jtd.2018.06.28

4. Williams TJ, Tuxen DV, Scheinkestel CD, et al. Risk factors for morbidity in mechanically ventilated patients with acute severe asthma. Am Rev Respir Dis. 1992;146:607-615. https://doi. org/10.1164/ajrccm/146.3.607 
5. Brenner B, Corbridge T, Kazzi A. Intubation and mechanical ventilation of the asthmatic patient in respiratory failure. Proc Am Thorac Soc. 2009;6:371-379. https://doi.org/10.1513/pats.P09ST4

6. Cho HJ, Kim DW, Kim GS, Jeong IS. anticoagulation therapy during extracorporeal membrane oxygenator support in pediatric patients. Chonnam Med J. 2017;53(2):110-117. https://doi. org/10.4068/cmj.2017.53.2.110

7. Chlebowski MM, Baltagi S, Carlson M, et al. Clinical controversies in anticoagulation monitoring and antithrombin supplementation for ECMO. Crit Care. 2020;24:19. https://doi.org/10.1186/ s13054-020-2726-9

8. Shoeb M, Fang MC. Assessing bleeding risk in patients taking anticoagulants. J Thromb Thrombolysis. 2013;35(3):312-319. https:// doi.org/10.1007/s11239-013-0899-7

9. Dang TO, Ogunniyi A, Barbee MS, Drilon A. Pembrolizumab for the treatment of PD-L1 positive advanced or metastatic non-small cell lung cancer. Expert Rev Anticancer Ther. 2016;16(1):13-20. https://doi.org/10.1586/14737140.2016.1123626

10. Bowman JD. The evolving role of PD-L1 testing in immunotherapy of metastatic melanoma and non-small cell lung cancer. $O B R$ Green. 2015;11(12):1-5.

How to cite this article: Klug TD, Craig J, Shires CB. Awake ECMO without anticoagulation for nearly completely obstructive squamous cell carcinoma of the trachea. Clin Case Rep. 2021;9:164-168. https://doi. org/10.1002/ccr3.3490 